\title{
MIXED APPROXIMATION OF EIGENVALUE PROBLEMS: A SUPERCONVERGENCE RESULT*
}

\author{
FRANCESCA GARDini ${ }^{1}$
}

\begin{abstract}
We state a superconvergence result for the lowest order Raviart-Thomas approximation of eigenvalue problems. It is known that a similar superconvergence result holds for the mixed approximation of Laplace problem; here we introduce a new proof, since the one given for the source problem cannot be generalized in a straightforward way to the eigenvalue problem. Numerical experiments confirm the superconvergence property and suggest that it also holds for the lowest order Brezzi-Douglas-Marini approximation.
\end{abstract}

Mathematics Subject Classification. 65N25, 65N30, 65Q60.

Received September 27, 2006. Revised December 19, 2008.

Published online April 8, 2009.

\section{INTRODUCTION}

This paper deals with a superconvergence result for mixed approximation of eigenvalue problems. It is well-known that a superconvergence property holds for the mixed approximation of Laplace problem (see [9]). Nevertheless, the proof given by Brezzi and Fortin [9] cannot be generalized in a easy way to eigenvalue problems. Indeed, the key point of the proof strongly relies on the Galerkin orthogonality, which holds for the source problem but not for the eigenvalue one.

In order to prove the superconvergence property we will use the equivalence between the lowest order Raviart-Thomas $\left(R T_{0}\right)$ approximation of Laplace eigenproblem with Neumann boundary conditions and the non-conforming piecewise linear Crouzeix-Raviart approximation (see [4]). We will also make use of a superconvergence result proved by Durán et al. in [11] for Laplace eigenproblem with Dirichlet boundary conditions.

An outline of the paper is as follows. Section 2 is devoted to the mathematical formulation of the model eigenvalue problem and to its mixed Raviart-Thomas and Brezzi-Douglas-Marini approximation. In Section 3 we recall the superconvergence result which holds for the source problem and we highlight the difficulties which arise changing from source to eigenvalue problem. In Section 4 we prove the superconvergence property by using the equivalence with a non-conforming approximation. Finally, in Section 5 we report the results of some numerical experiments which confirm the superconvergence property. Furthermore, numerical computations suggest that the superconvergence property holds for the lowest order Brezzi-Douglas-Marini $\left(B D M_{1}\right)$ space as well.

\footnotetext{
Keywords and phrases. Eigenvalue problem, mixed finite element, superconvergence result.

* This work was partially supported by the IMATI (CNR-Pavia, Italy).

${ }^{1}$ Dipartimento di Matematica "F. Casorati", Università di Pavia, 27100 Pavia, Italy. francesca.gardini@unipv.it
} 


\section{StATEMENT OF THE PROBLEM AND ITS DisCRETIZATION}

Let $\Omega \subset \mathbb{R}^{d}(d=2,3)$ be a simply connected polygonal or polyhedral domain. We consider the following eigenvalue problem:

$$
\begin{aligned}
& \text { Find } \lambda \in \mathbb{R} \text { s.t. } \\
& \begin{cases}-\Delta \varphi=\lambda \varphi & \text { in } \Omega \\
\frac{\partial \varphi}{\partial \boldsymbol{n}}=0 & \text { on } \partial \Omega,\end{cases}
\end{aligned}
$$

where $\boldsymbol{n}$ denotes the outward normal unit vector.

For the sake of simplicity we shall develop the analysis in two dimensions, being the extension to three dimensions straightforward.

We shall use the standard notation for the Sobolev spaces $H^{m}(\Omega)$, their norms $\|\cdot\|_{m}$, and seminorms $|\cdot|_{m}$ (see [1]). As usual we denote by $(\cdot, \cdot)$ the $L^{2}$-inner product.

Introducing $\boldsymbol{\sigma}=\nabla \varphi$, we obtain the usual mixed formulation of problem (1.1) which in weak form is given by

$$
\begin{aligned}
& \text { Find } \lambda \in \mathbb{R} \text { s.t. there exists }(\boldsymbol{\sigma}, \varphi) \in H_{0}(\operatorname{div}, \Omega) \times L_{0}^{2}(\Omega), \text { with } \varphi \neq 0 \text { : } \\
& \begin{cases}(\boldsymbol{\sigma}, \boldsymbol{\tau})+(\operatorname{div} \boldsymbol{\tau}, \varphi)=0 & \forall \boldsymbol{\tau} \in H_{0}(\operatorname{div}, \Omega) \\
(\operatorname{div} \boldsymbol{\sigma}, \psi)=-\lambda(\varphi, \psi) & \forall \psi \in L_{0}^{2}(\Omega),\end{cases}
\end{aligned}
$$

where $L_{0}^{2}(\Omega)$ is the space consisting of square Lebesgue-integrable functions having zero mean value and

$$
H_{0}(\operatorname{div}, \Omega)=\left\{\boldsymbol{v} \in L^{2}(\Omega)^{2}: \operatorname{div} \boldsymbol{v} \in L^{2}(\Omega) \text { and } \boldsymbol{v} \cdot \boldsymbol{n}=\mathbf{0} \text { on } \partial \Omega\right\}
$$

is endowed with the usual norm $\|\boldsymbol{v}\|_{\text {div }}^{2}=\|\boldsymbol{v}\|_{0}^{2}+\|\operatorname{div} \boldsymbol{v}\|_{0}^{2}$. Here and thereafter conditions of the type $v=0$ on $\partial \Omega$ are to be understood in the sense of traces (see [16]).

It is well-known that problem (1.2) admits a countable set of real and positive eigenvalues, which can be ordered in an increasing divergent sequence and the corresponding eigenfunctions give rise to an orthonormal basis of $L^{2}(\Omega)^{2}$. Moreover each eigenspace is finite dimensional. Finally, due to regularity results (see [15]), there exists a constant $s>1 / 2$ (depending on $\Omega$ ), such that $(\sigma, \varphi)$ belongs to the space $H^{s}(\Omega)^{2} \times H^{1+s}(\Omega)$. Furthermore the following estimate holds true:

$$
\|\boldsymbol{\sigma}\|_{s}+\|\operatorname{div} \boldsymbol{\sigma}\|_{1+s} \leq C\|\boldsymbol{\sigma}\|_{0}
$$

where $C$ is a constant depending on the eigenvalue $\lambda$. In (1.3) $s$ is at least one if $\Omega$ is convex, while $s$ is at least $\pi / \omega-\varepsilon$ for any $\varepsilon>0$ for a non convex domain, $\omega<2 \pi$ being the maximum interior angle of $\Omega$.

Let $\left\{\mathscr{T}_{h}\right\}$ denote a shape-regular family (i.e., satisfying the minimum angle condition, see [10]) of simplicial decomposition of $\Omega$. As usual we require that any two elements in $\mathscr{T}_{h}$ share at most a common edge or a common vertex, and we denote by $h$ the maximum diameter of the elements $K$ in $\mathscr{T}_{h}$.

Let $\Sigma_{h}$ and $\Phi_{h}$ denote two finite dimensional subspaces of $H_{0}(\operatorname{div}, \Omega)$ and $L_{0}^{2}(\Omega)$, respectively. The discretization of problem (1.2) is then given by

$$
\begin{aligned}
& \text { Find } \lambda_{h} \in \mathbb{R} \text { s.t. there exists }\left(\boldsymbol{\sigma}_{\boldsymbol{h}}, \varphi_{h}\right) \in \Sigma_{h} \times \Phi_{h}, \text { with } \varphi_{h} \neq 0 \text { : } \\
& \begin{cases}\left(\boldsymbol{\sigma}_{h}, \boldsymbol{\tau}\right)+\left(\operatorname{div} \boldsymbol{\tau}, \varphi_{h}\right)=0 & \forall \boldsymbol{\tau} \in \Sigma_{h} \\
\left(\operatorname{div} \boldsymbol{\sigma}_{h}, \psi\right)=-\lambda_{h}\left(\varphi_{h}, \psi\right) & \forall \psi \in \Phi_{h} .\end{cases}
\end{aligned}
$$

Usual spaces used to approximate $H(\operatorname{div}, \Omega)$ are Raviart-Thomas and Brezzi-Douglas-Marini ones. In what follows we recall their definitions for triangular decompositions of $\Omega$ (see [7] for general quadrilateral meshes) and their main properties. 
The Raviart-Thomas space of order $k(k \geq 0)$ is defined (see [9]) by

$$
R T_{k}=\left\{\boldsymbol{\tau} \in H(\operatorname{div}, \Omega):\left.\boldsymbol{\tau}\right|_{K}\left(x_{1}, x_{2}\right)=\mathbb{P}_{k}(K)^{2}+\left(x_{1}, x_{2}\right) \mathbb{P}_{k}(K)\right\}, \quad \forall K \in \mathscr{T}_{h}
$$

where $\mathbb{P}_{k}(K)$ denotes the space of polynomials of degree at most $k$ on $K$.

The mixed Raviart-Thomas approximation of problem (1.2) is then obtained taking

$$
\Sigma_{h}=H_{0}(\operatorname{div}, \Omega) \cap R T_{k}
$$

and

in (1.4).

$$
\Phi_{h}=\left\{\psi \in L_{0}^{2}(\Omega):\left.\psi\right|_{K} \in \mathbb{P}_{k}(K) \quad \forall K \in \mathscr{T}_{h}\right\}
$$

As usual using the degrees of freedom it is possible to define an interpolation operator $\Pi_{h} \tau$, provided $\tau$ is slightly smoother than merely belonging to $H(\operatorname{div}, \Omega)$, i.e. $\boldsymbol{\tau} \in H(\operatorname{div}, \Omega) \cap H^{\varepsilon}(\Omega)$, for some $\varepsilon>0$ (see [9]). Then the following commuting diagram holds:

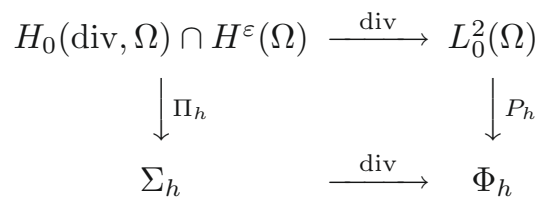

where $P_{h}$ denotes the $L^{2}$-projection on $\Phi_{h}$.

The following theorem states the approximation properties of Raviart-Thomas finite element spaces.

Theorem 1.1. Given any vectorfield $\boldsymbol{u}$ smooth enough such that the right hand side of the next expression makes sense, there exists $\boldsymbol{u}^{\mathrm{I}}=\Pi_{h} \boldsymbol{u} \in V_{h}$ such that

$$
\left\|\boldsymbol{u}-\boldsymbol{u}^{\mathrm{I}}\right\|_{\operatorname{div}} \leq C h^{k+1}\left(|\boldsymbol{u}|_{k+1}+|\operatorname{div} \boldsymbol{u}|_{k+1}\right) .
$$

The Brezzi-Douglas-Marini space of order $k(k \geq 1)$ is defined (see [9]) by

$$
B D M_{k}=\left\{\boldsymbol{\tau} \in H(\operatorname{div}, \Omega):\left.\boldsymbol{\tau}\right|_{K} \in \mathbb{P}_{k}(K)^{2} \quad \forall K \in \mathscr{T}_{h}\right\}
$$

Then the Brezzi-Douglas-Marini mixed approximation of problem (1.2) is obtained taking

$$
\Sigma_{h}=B D M_{k} \cap H_{0}(\operatorname{div}, \Omega)
$$

and

in (1.4).

$$
\Phi_{h}=\left\{\psi \in L_{0}^{2}(\Omega):\left.\psi\right|_{K} \in \mathbb{P}_{k-1}(K) \forall K \in \mathscr{T}_{h}\right\}
$$

Notice that the commuting diagram property (1.6) holds true for $B D M$ elements as well.

Finally, the following theorem states the approximation properties of Brezzi-Douglas-Marini finite element spaces.

Theorem 1.2. Given any vectorfield $\boldsymbol{u}$ smooth enough such that the right hand side of the next expressions makes sense, there exists $\boldsymbol{u}^{\mathrm{I}}=\Pi_{h} \boldsymbol{u} \in \Sigma_{h}$ such that

$$
\left\|\boldsymbol{u}-\boldsymbol{u}^{\mathrm{I}}\right\|_{0} \leq C h^{k+1}|\boldsymbol{u}|_{k+1}
$$

and

$$
\left\|\operatorname{div}\left(\boldsymbol{u}-\boldsymbol{u}^{\mathrm{I}}\right)\right\|_{0} \leq C h^{k}|\operatorname{div} \boldsymbol{u}|_{k}
$$


Assume for simplicity that $\lambda$ is a simple eigenvalue of problem $(1.2)$, with $(\boldsymbol{\sigma}, \varphi)$ its corresponding eigenfunction. Let $\left(\lambda_{h}, \boldsymbol{\sigma}_{h}\right)$ denote the $R T(B D M)$ approximation to $(\lambda, \boldsymbol{\sigma})$ and let us assume that $\|\boldsymbol{\sigma}\|_{0}=\left\|\boldsymbol{\sigma}_{h}\right\|_{0}=1$. Then, it follows from the abstract theory (see $[5,6]$ ) and known a priori error estimates that for $h$ small enough (depending on $\lambda$ ),

$$
\begin{aligned}
\left\|\boldsymbol{\sigma}-\boldsymbol{\sigma}_{h}\right\|_{\text {div }} & \leq C h^{t} \\
\left\|\boldsymbol{\sigma}-\boldsymbol{\sigma}_{h}\right\|_{0} & \leq C h^{r} \\
\left|\lambda-\lambda_{h}\right| & \leq C h^{2 t},
\end{aligned}
$$

where $r=\min \{k+1, s\}$, and $t=\min \{s, k\}$ if $\Sigma_{h}$ is the Brezzi-Douglas-Marini space, otherwise if $\Sigma_{h}$ is the Raviart-Thomas space then $t=r=\min \{k+1, s\}$.

Remark 1.3. If the eigenfunction $\boldsymbol{\sigma}$ is smooth enough (i.e. belongs to the space $H(\operatorname{div}, \Omega) \cap H^{\alpha}(\Omega)$ for some $\alpha>1$ ) then, contrary to $R T$ elements, $B D M$ ones provide a $L^{2}$-approximation of higher order than the $H(\operatorname{div})$ approximation.

\section{Source PRoblem}

In this section we recall the superconvergence property which holds for the source problem associated with Laplace eigenproblem, and we highlight why the proof cannot be generalized in a trivial way to show the analogous result for the eigenvalue problem. For general superconvergence results for Laplace problem and possible applications to a posteriori error estimation we refer to $[8,9]$ and the references therein.

The standard mixed formulation of Laplace problem with Neumann boundary conditions reads as follows:

$$
\begin{aligned}
& \text { Given } f \in L_{0}^{2}(\Omega) \text {, find }\left(\boldsymbol{\sigma}^{*}, \varphi^{*}\right) \in H_{0}(\operatorname{div}, \Omega) \times L_{0}^{2}(\Omega) \text { : } \\
& \begin{cases}\left(\boldsymbol{\sigma}^{*}, \boldsymbol{\tau}\right)+\left(\operatorname{div} \boldsymbol{\tau}, \varphi^{*}\right)=0 & \forall \boldsymbol{\tau} \in H_{0}(\operatorname{div}, \Omega) \\
\left(\operatorname{div} \boldsymbol{\sigma}^{*}, \psi\right)=-(f, \psi) & \forall \psi \in L_{0}^{2}(\Omega) .\end{cases}
\end{aligned}
$$

It is well known that problem (2.1) is well-posed. Moreover, due to regularity results (see [15]), there exists a constant $s>1 / 2$ (depending on $\Omega$ ), such that $\left(\boldsymbol{\sigma}^{*}, \varphi^{*}\right)$ belongs to the space $H^{s}(\Omega)^{2} \times H^{1+s}(\Omega)$. Finally, the following stability estimate holds true:

$$
\left\|\boldsymbol{\sigma}^{*}\right\|_{s}+\left\|\varphi^{*}\right\|_{1+s} \leq C\|f\|_{0} .
$$

We only consider Raviart-Thomas approximation of problem (2.1), since the analysis can be extended to Brezzi-Douglas-Marini ones in a straightforward way. The discrete problem is then given by

$$
\begin{aligned}
& \text { Given } f \in L_{0}^{2}(\Omega) \text {, find }\left(\boldsymbol{\sigma}_{h}^{*}, \varphi_{h}^{*}\right) \in \Sigma_{h} \times \Phi_{h} \text { : } \\
& \begin{cases}\left(\boldsymbol{\sigma}_{h}^{*}, \boldsymbol{\tau}\right)+\left(\operatorname{div} \boldsymbol{\tau}, \varphi_{h}^{*}\right)=0 & \forall \boldsymbol{\tau} \in \Sigma_{h} \\
\left(\operatorname{div} \boldsymbol{\sigma}_{h}^{*}, \psi\right)=-(f, \psi) & \forall \psi \in \Phi_{h},\end{cases}
\end{aligned}
$$

where $\Sigma_{h}$ is the Raviart-Thomas finite element space of order $k$ and

$$
\Phi_{h}=\left\{\psi \in L_{0}^{2}(\Omega):\left.\psi\right|_{K} \in \mathbb{P}_{k} \forall K \in \mathscr{T}_{h}\right\} .
$$

It is well known that problem (2.3) is well-posed as well. Moreover, it follows from the standard a priori error analysis for mixed problems that there exists a positive constant $C$, independent of $h$ such that the following estimate holds:

$$
\left\|\boldsymbol{\sigma}^{*}-\boldsymbol{\sigma}_{h}^{*}\right\|_{\operatorname{div}} \leq C h^{\min \{s, k+1\}}\left(\left|\boldsymbol{\sigma}^{*}\right|_{s}+\left|\operatorname{div} \boldsymbol{\sigma}^{*}\right|_{s}\right) .
$$

Then the following superconvergence result holds true (see [9]). 
Theorem 2.1. $\left\|P_{h} \varphi^{*}-\varphi_{h}^{*}\right\|_{0}$ is a higher-order term than $\left\|\boldsymbol{\sigma}^{*}-\boldsymbol{\sigma}_{h}^{*}\right\|_{\text {div }}$.

Proof. Let $(\boldsymbol{z}, \phi)$ denote the solution of the following auxiliary problem:

$$
\begin{aligned}
& \text { Find }(\boldsymbol{z}, \phi) \in H_{0}(\operatorname{div}, \Omega) \times L_{0}^{2}(\Omega): \\
& \begin{cases}(\boldsymbol{\tau}, \boldsymbol{z})+(\operatorname{div} \boldsymbol{\tau}, \phi)=0 & \forall \boldsymbol{\tau} \in H_{0}(\operatorname{div}, \Omega) \\
(\operatorname{div} \boldsymbol{z}, \psi)=\left(P_{h} \varphi^{*}-\varphi_{h}^{*}, \psi\right) & \forall \psi \in L_{0}^{2}(\Omega) .\end{cases}
\end{aligned}
$$

Then taking into account the commuting diagram property (1.6) together with the definition of $P_{h}$ and the Galerkin orthogonality property relative to problems (2.1) and (2.3), it is possible to estimate $\left\|P_{h} \varphi^{*}-\varphi_{h}^{*}\right\|_{0}$ in the following way:

$$
\begin{aligned}
\left\|P_{h} \varphi^{*}-\varphi_{h}^{*}\right\|_{0}^{2} & =\left(\operatorname{div} \boldsymbol{z}, P_{h} \varphi^{*}-\varphi_{h}^{*}\right)=\left(\operatorname{div} \Pi_{h} \boldsymbol{z}, P_{h} \varphi^{*}-\varphi_{h}^{*}\right) \\
& =\left(\operatorname{div} \Pi_{h} \boldsymbol{z}, \varphi^{*}-\varphi_{h}^{*}\right)=-\left(\boldsymbol{\sigma}^{*}-\boldsymbol{\sigma}_{h}^{*}, \Pi_{h} \boldsymbol{z}\right) \\
& =\left(\boldsymbol{\sigma}^{*}-\boldsymbol{\sigma}_{h}^{*}, \boldsymbol{z}-\Pi_{h} \boldsymbol{z}\right)-\left(\boldsymbol{\sigma}^{*}-\boldsymbol{\sigma}_{h}^{*}, \boldsymbol{z}\right) \\
& =\left(\boldsymbol{\sigma}^{*}-\boldsymbol{\sigma}_{h}^{*}, \boldsymbol{z}-\Pi_{h} \boldsymbol{z}\right)+\left(\operatorname{div}\left(\boldsymbol{\sigma}^{*}-\boldsymbol{\sigma}_{h}^{*}\right), \phi\right) \\
& =\left(\boldsymbol{\sigma}^{*}-\boldsymbol{\sigma}_{h}^{*}, \boldsymbol{z}-\Pi_{h} \boldsymbol{z}\right)+\left(\operatorname{div}\left(\boldsymbol{\sigma}^{*}-\boldsymbol{\sigma}_{h}^{*}\right), \phi-P_{h} \phi\right) .
\end{aligned}
$$

The first term in the right hand side of the above equation can be estimated using the Cauchy-Schwartz inequality and the interpolation error estimate (1.7) as follows:

$$
\left(\boldsymbol{\sigma}^{*}-\boldsymbol{\sigma}_{h}^{*}, \boldsymbol{z}-\Pi_{h} \boldsymbol{z}\right) \leq C h^{\min \{k+1, s\}}|\boldsymbol{z}|_{s}\left\|\boldsymbol{\sigma}^{*}-\boldsymbol{\sigma}_{h}^{*}\right\|_{0}
$$

The second term instead is bounded above using the $L^{2}$-projection error estimate

$$
\left(\operatorname{div}\left(\boldsymbol{\sigma}^{*}-\boldsymbol{\sigma}_{h}^{*}\right), \phi-P_{h} \phi\right) \leq C h^{\min \{k+1,1+s\}}|\phi|_{1+s}\left\|\operatorname{div}\left(\boldsymbol{\sigma}^{*}-\boldsymbol{\sigma}_{h}^{*}\right)\right\|_{0}
$$

Putting together the above inequalities, and taking into account the stability estimate (2.2), we get

$$
\left\|P_{h} \varphi^{*}-\varphi_{h}^{*}\right\|_{0} \leq C h^{\min \{k+1, s\}}\left\|\boldsymbol{\sigma}^{*}-\boldsymbol{\sigma}_{h}^{*}\right\|_{0}+C h^{\min \{k+1,1+s\}}\left\|\operatorname{div}\left(\boldsymbol{\sigma}^{*}-\boldsymbol{\sigma}_{h}^{*}\right)\right\|_{0}
$$

and hence

$$
\left\|P_{h} \varphi^{*}-\varphi_{h}^{*}\right\|_{0} \leq C h^{\alpha}\left\|\sigma^{*}-\sigma_{h}^{*}\right\|_{\text {div }}
$$

where $\alpha$ is a strictly positive constant.

Remark 2.2. Since $\left\|\operatorname{div}\left(\boldsymbol{\sigma}^{*}-\boldsymbol{\sigma}_{h}^{*}\right)\right\|_{0}$ and $\left\|\varphi^{*}-\varphi_{h}^{*}\right\|_{0}$ are of the same order as $\left\|\boldsymbol{\sigma}^{*}-\boldsymbol{\sigma}_{h}^{*}\right\|_{0},\left\|P_{h} \varphi^{*}-\varphi_{h}^{*}\right\|_{0}$ turns out to be higher order than $\left\|\boldsymbol{\sigma}^{*}-\boldsymbol{\sigma}_{h}^{*}\right\|_{0}$ and $\left\|\varphi^{*}-\varphi_{h}^{*}\right\|_{0}$ as well.

Remark 2.3. We observe that the proof of Theorem 2.1 can be generalized without any change to BrezziDouglas-Marini approximation. Moreover, since $h\left\|\operatorname{div}\left(\boldsymbol{\sigma}^{*}-\boldsymbol{\sigma}_{h}^{*}\right)\right\|_{0}$ is at least of the same order as $\left\|\boldsymbol{\sigma}^{*}-\boldsymbol{\sigma}_{h}^{*}\right\|_{0}$ and $\left\|\sigma^{*}-\sigma_{h}^{*}\right\|_{0}$ is at least of the same order as $\left\|\varphi^{*}-\varphi_{h}^{*}\right\|_{0}$, Remark 2.2 holds as well.

We end this section observing that the proof of the superconvergence property cannot be straightforwardly extended to the eigenvalue problem. In fact in this case the Galerkin orthogonality does not hold and hence we are not allowed to subtract $P_{h} \phi$ in the last step of equation (2.6).

\section{Eigenvalue PROBlem}

In this section we extend the superconvergence result stated in Theorem 2.1 to the eigenvalue problem using the equivalence between the lowest order Raviart-Thomas approximation and the non-conforming piecewise 
linear Crouzeix-Raviart approximation enriched by local bubbles. This alternative finite element formulation has been also used in the context of elastoacoustics vibration problem to obtain an accurate pressure post-process (see [3]).

Following the arguments given in $[4,17]$, it can be seen that the lowest order Raviart-Thomas approximation of problem (1.2) is equivalent to a nonconforming approximation of the standard formulation of (1.1). Let us introduce the nonconforming space of Crouzeix and Raviart enriched by local bubbles. Denoting by $\mathcal{B}(K)$ the space of cubic polynomials vanishing on $\partial K$, we define

$$
\begin{aligned}
X_{h} & =\left\{\phi \in L^{2}(\Omega):\left.\phi\right|_{K} \in \mathbb{P}_{1}(K) \forall K \in \mathscr{T}_{h}, \phi \text { is continuous at midpoints of interior interfaces }\right\} \\
B_{h} & =\left\{b \in H_{0}^{1}(\Omega):\left.b\right|_{K} \in \mathcal{B}(K) \forall K \in \mathscr{T}_{h}\right\} \\
W_{h} & =X_{h} \bigoplus B_{h}
\end{aligned}
$$

Let

$$
\Sigma_{h}^{d}=\left\{\boldsymbol{v} \in L^{2}(\Omega)^{2}:\left.\boldsymbol{v}\right|_{K} \in R T_{0}(K) \quad \forall K \in \mathscr{T}_{h}\right\} .
$$

We also introduce the following $L^{2}$-projection operator:

$$
\begin{aligned}
& P_{\Sigma_{h}^{d}}: L^{2}(\Omega)^{2} \longrightarrow \Sigma_{h}^{d} \\
& P_{\Sigma_{h}^{d}} \boldsymbol{v} \in \Sigma_{h}^{d} \text { such that }\left(\boldsymbol{v}-P_{\Sigma_{h}^{d}} \boldsymbol{v}, \boldsymbol{v}_{h}\right)=0 \quad \forall \boldsymbol{v}_{h} \in \Sigma_{h}^{d} .
\end{aligned}
$$

In the following we denote by $\nabla_{h} \psi_{h}$ the elementwise gradient of $\psi_{h}$. Then problem (1.4) is equivalent to the following one:

Find $\lambda_{h} \in \mathbb{R}$ s.t. there exists $\phi_{h} \in W_{h}$, with $\phi_{h} \neq 0$ s.t.

$$
\left(P_{\Sigma_{h}^{d}}\left(\nabla_{h} \phi_{h}\right), \nabla_{h} \psi_{h}\right)=\lambda_{h}\left(P_{h} \phi_{h}, \psi_{h}\right) \quad \forall \psi_{h} \in W_{h},
$$

in the sense that they have the same eigenvalues $\lambda_{h}$ and the eigenvectors are related, up to a constant, by $\boldsymbol{\sigma}_{h}=P_{\Sigma_{h}^{d}}\left(\nabla_{h} \phi_{h}\right)$ and $\varphi_{h}=P_{h} \phi_{h}($ see $[4,17])$.

Applying the general theory developed in [5], Durán et al. in [11] proved (for Dirichlet boundary conditions) the following result:

$$
\left\|\varphi-\bar{\phi}_{h}\right\|_{0}=O\left(h^{2 t}\right)
$$

where $\bar{\phi}_{h}$ is a multiple of $\phi_{h}$ such that $\left\|\bar{\phi}_{h}\right\|_{0}=\|\varphi\|_{0}$ and $t=\min \{1, s\}$. As stated in [2], the above result can be extended to the case of Neumann boundary conditions.

We now state a result which is useful in the proof of the superconvergence property.

Lemma 3.1. If $\left(\lambda_{h}, \phi_{h}\right)$ is an eigensolution of problem (3.3), then the elementwise $H^{1}-$ seminorm $\left|\phi_{h}\right|_{1, h}$ is bounded.

Proof. First of all we notice that, since $\phi_{h}$ is an eigensolution of problem (3.3), it holds

$$
\left\|P_{\Sigma_{h}^{d}}\left(\nabla_{h} \phi_{h}\right)\right\|_{0}^{2}=\lambda_{h}\left\|P_{h} \phi_{h}\right\|_{0}^{2} \leq \lambda_{h}\left\|\phi_{h}\right\|_{0}^{2}
$$

Since $\phi_{h} \in W_{h}$ it can be written as $\phi_{h}=p_{h}+b_{h}$, with $p_{h} \in C R_{h}$ and $b_{h} \in B_{h}$. Observing that $\int_{\Omega} \nabla_{h} p_{h} \cdot \nabla b_{h}=0$ and $P_{\Sigma_{h}^{d}}\left(\nabla_{h} p_{h}\right)=\nabla_{h} p_{h}$, we obtain

and

$$
\left\|\nabla_{h} \phi_{h}\right\|_{0}^{2}=\left\|\nabla_{h} p_{h}\right\|_{0}^{2}+\left\|\nabla b_{h}\right\|_{0}^{2}
$$

$$
\left\|P_{\Sigma_{h}^{d}}\left(\nabla_{h} \phi_{h}\right)\right\|_{0}^{2}=\left\|\nabla_{h} p_{h}\right\|_{0}^{2}+\left\|P_{\Sigma_{h}^{d}}\left(\nabla b_{h}\right)\right\|_{0}^{2}
$$

Hence it is enough to prove that $\left\|\nabla b_{h}\right\|_{0}^{2}$ is bounded. In particular, we shall prove that there exists a constant $C$ independent of $h$ such that $\left\|\nabla b_{h}\right\|_{0} \leq C\left\|P_{\Sigma_{h}}\left(\nabla b_{h}\right)\right\|_{0}$. 
We choose as basis of the space $\Sigma_{h}^{d}$ the one given on each element $K$ by

$$
(1,0)^{t} \quad(0,1)^{t} \quad \boldsymbol{x}-\boldsymbol{x}_{c}
$$

where $\boldsymbol{x}=(x, y)$ and $\boldsymbol{x}_{c}$ are the coordinates of the barycenter of $K$. Then it can be easily checked that

$$
\left.\left(P_{\Sigma_{h}} \nabla b_{h}\right)\right|_{K}=\frac{-2 \int_{K} b_{h, K}}{\int_{K}\left|\boldsymbol{x}-\boldsymbol{x}_{c}\right|^{2}}\left(\boldsymbol{x}-\boldsymbol{x}_{c}\right)
$$

where $b_{h, K}=\left.b_{h}\right|_{K}$. From (3.5) it follows that $\left.\left(P_{\Sigma_{h}} \nabla b_{h}\right)\right|_{K}=0$ if and only if $b_{h, K}=0$, equivalently $\nabla b_{h, K}=0$. Therefore, there exists a constant $C$ such that $\left\|\nabla b_{h, K}\right\|_{0, K} \leq C\left\|P_{\Sigma_{h}}\left(\nabla b_{h}\right)\right\|_{0, K}$. Finally, since $\left\|\nabla b_{h, K}\right\|_{0}$ and $\left\|P_{\Sigma_{h}^{d}}\left(\nabla b_{h, K}\right)\right\|_{0}$ scales in the same way, it turns out that the constant $C$ is independent of the mesh size $h$.

Then, the following result which generalise to eigenvalue problem the superconvergence property holds true:

Theorem 3.2. Let $(\lambda, \varphi)$ be the eigensolution of Laplace eigenproblem (1.1) and $\left(\lambda_{h}, \varphi_{h}\right)$ be the corresponding discrete eigenpair of problem (1.4). Then it holds

$$
\left\|P_{h} \varphi-\varphi_{h}\right\|_{0}=O\left(h^{2 t}\right),
$$

where $t=\min \{1, s\}$.

Proof. Since the eigenfunctions are defined up to a constant, we choose $\boldsymbol{\sigma}$ and $\boldsymbol{\sigma}_{\boldsymbol{h}}$ such that $\|\boldsymbol{\sigma}\|_{0}=1$ and $\left\|\boldsymbol{\sigma}_{\boldsymbol{h}}\right\|_{0}=1$. Then it holds

and

$$
\|\varphi\|_{0}=\frac{1}{\sqrt{\lambda}}
$$

$$
\left\|\varphi_{h}\right\|_{0}=\frac{1}{\sqrt{\lambda_{h}}}
$$

Let $\bar{\phi}_{h}$ be the eigenvector of problem (3.3) corresponding to the eigenvalue $\lambda_{h}$ and satisfying $\left\|\bar{\phi}_{h}\right\|_{0}=\|\varphi\|_{0}$. Then thanks to the equivalence between the lowest order Raviart-Thomas approximation of problem (1.2) and (3.3),

$$
\varphi_{h}=\frac{1}{\sqrt{\lambda_{h}}} \frac{P_{h} \bar{\phi}_{h}}{\left\|P_{h} \bar{\phi}_{h}\right\|_{0}} .
$$

Moreover, since the projection $P_{h}$ is a contraction, we have

$$
\left\|P_{h} \varphi-\varphi_{h}\right\|_{0}=\left\|P_{h} \varphi-\frac{1}{\sqrt{\lambda_{h}}} \frac{P_{h} \bar{\phi}_{h}}{\left\|P_{h} \bar{\phi}_{h}\right\|_{0}}\right\|_{0} \leq\left\|\varphi-\frac{1}{\sqrt{\lambda_{h}}} \frac{\bar{\phi}_{h}}{\left\|P_{h} \bar{\phi}_{h}\right\|_{0}}\right\|_{0} \leq\left\|\varphi-\bar{\phi}_{h}\right\|_{0}+\left\|\bar{\phi}_{h}-\frac{1}{\sqrt{\lambda_{h}}} \frac{\bar{\phi}_{h}}{\left\|P_{h} \bar{\phi}_{h}\right\|_{0}}\right\|_{0} .
$$

The first term in the right hand side of the above equation is $O\left(h^{2 t}\right)$, as we have said in (3.4). Hence, it remains to prove that

This follows from the fact that

$$
\left\|\bar{\phi}_{h}-\frac{1}{\sqrt{\lambda_{h}}} \frac{\bar{\phi}_{h}}{\left\|P_{h} \bar{\phi}_{h}\right\|_{0}}\right\|_{0}=O\left(h^{2 t}\right) .
$$

$$
\left\|\bar{\phi}_{h}\right\|_{0}-\left\|P_{h} \bar{\phi}_{h}\right\|_{0}=O\left(h^{2}\right),
$$

and

Indeed

$$
\left|\frac{1}{\sqrt{\lambda}}-\frac{1}{\sqrt{\lambda_{h}}}\right|=O\left(h^{2 t}\right) .
$$

$$
\left\|\bar{\phi}_{h}-\frac{1}{\sqrt{\lambda_{h}}} \frac{\bar{\phi}_{h}}{\left\|P_{h} \bar{\phi}_{h}\right\|_{0}}\right\|_{0}=\left\|\bar{\phi}_{h}\right\|_{0}\left|\frac{\left\|P_{h} \bar{\phi}_{h}\right\|_{0} \sqrt{\lambda_{h}}-1}{\left\|P_{h} \bar{\phi}_{h}\right\|_{0} \sqrt{\lambda_{h}}}\right|,
$$


and

$$
\left|\left\|P_{h} \bar{\phi}_{h}\right\|_{0} \sqrt{\lambda_{h}}-1\right| \leq \sqrt{\lambda_{h}}\left(\left|\left\|\bar{\phi}_{h}\right\|_{0}-\left\|P_{h} \bar{\phi}_{h}\right\|_{0}\right|+\left|\frac{1}{\sqrt{\lambda}}-\frac{1}{\sqrt{\lambda_{h}}}\right|\right) .
$$

We now prove (3.7). From the a priori error estimate (1.12) it follows

$$
\left|\frac{1}{\sqrt{\lambda}}-\frac{1}{\sqrt{\lambda_{h}}}\right|=\frac{\left|\lambda-\lambda_{h}\right|}{\lambda_{h} \sqrt{\lambda}+\lambda \sqrt{\lambda_{h}}}=O\left(h^{2 t}\right) .
$$

Finally,

$$
\left\|\bar{\phi}_{h}\right\|_{0}-\left\|P_{h} \bar{\phi}_{h}\right\|_{0} \leq\left\|\bar{\phi}_{h}-P_{h} \bar{\phi}_{h}\right\|_{0} \leq C h
$$

where the last inequality follows from the $L^{2}$-projection error estimate and from the fact that the broken $H^{1}$-seminorm $\left|\bar{\phi}_{h}\right|_{1, h}$ is bounded as stated in Proposition 3.1. Then (3.6) easily follows since

$$
\left\|\bar{\phi}_{h}\right\|_{0}-\left\|P_{h} \bar{\phi}_{h}\right\|_{0}=\frac{\left\|\bar{\phi}_{h}\right\|_{0}^{2}-\left\|P_{h} \bar{\phi}_{h}\right\|_{0}^{2}}{\left\|\bar{\phi}_{h}\right\|_{0}+\left\|P_{h} \bar{\phi}_{h}\right\|_{0}}=\frac{\left\|\bar{\phi}_{h}-P_{h} \bar{\phi}_{h}\right\|_{0}^{2}}{\left\|\bar{\phi}_{h}\right\|_{0}+\left\|P_{h} \bar{\phi}_{h}\right\|_{0}}=O\left(h^{2}\right)
$$

Remark 3.3. From Theorem 3.2 together with the a priori error estimate (1.10), we get that $\left\|P_{h} \varphi-\varphi_{h}\right\|_{0}$ is of higher order than $\left\|\varphi-\varphi_{h}\right\|_{0}$ and $\left\|\boldsymbol{\sigma}-\boldsymbol{\sigma}_{h}\right\|_{0}$.

\section{NumericAl RESULTS}

In this section we present the results of some numerical experiments which fully confirm the superconvergence property stated in the previous section. Moreover, we investigate numerically whether or not the superconvergence property holds for $B D M_{1}$ elements as well.

Numerical computations directed to investigate whether or not the superconvergence property holds for higher order Raviart-Thomas and Brezzi-Douglas-Marini elements as well are still in progress.

\subsection{Square domain}

As a first example we consider the domain $\Omega=(0, \pi) \times(0, \pi)$. In this case the eigensolutions of Laplace eigenproblem with homogeneous Neumann boundary conditions are known and are given by eigenvalues

$$
\lambda=n^{2}+m^{2},
$$

with corresponding eigenfunctions

$$
\varphi=\cos (n x) \cos (m y),
$$

where $n, m \in \mathbb{N}$ are not simultaneously vanishing.

We choose as exact eigenpair $(\lambda, \varphi)=(2, \cos x \cos y)$ and we use $R T_{0}$ and $B D M_{1}$ as approximation spaces.

We test the superconvergence property on two sequences of meshes, both structured and unstructured as shown in Figures 1 and 2. The meshes are obtained from an initial triangulation of the square by regular refinement, namely subdividing each triangle by joining the midpoints of each edge.

The first test concerns $R T_{0}$ approximation. In Tables 1 and 2 we report both the $L^{2}$ and the $H$ (div)-norms of the error in the approximation of the eigenfunction and we compute the numerical rate of convergence, which is 1 as predicted by the a priori error estimates (1.10) and (1.11) for regular eigenmodes. We also report the $L^{2}$-norm of the error between the projection of the continuous eigenfunction and the discrete one. In this case the order of convergence is 2, as predicted by Theorem 3.2 for regular eigenmodes. Eventually, in Figure 3 we plot the above errors using a $\log / \log$ scale.

In the second test we consider $B D M_{1}$ finite elements. The results of these experiments are shown in Tables 3 and 4. As predicted by the a priori error estimates (1.10) and (1.11) for regular eigenmodes, the order of convergence is 1 in the $H$ (div)-norm and 2 in the $L^{2}$-norm. Moreover, the numerical results suggest that the superconvergence property holds as well. Finally, in Figure 4 we plot the errors in a $\log / \log$ scale. 

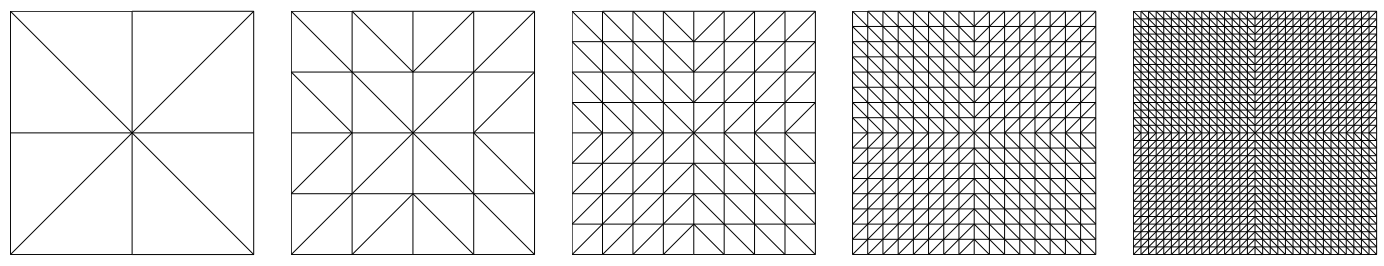

FiguRE 1. Structured meshes.
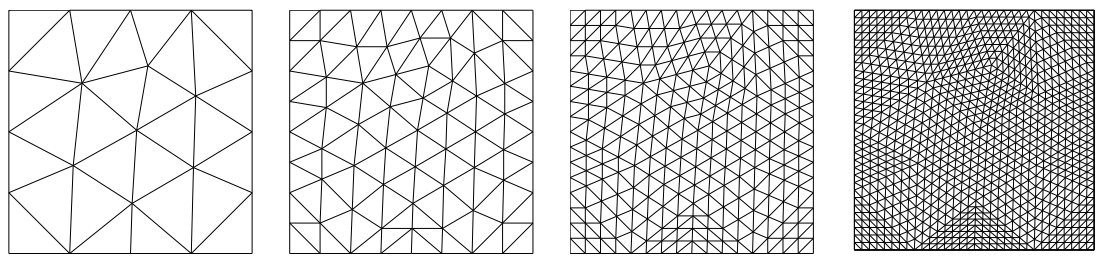

Figure 2. Unstructured meshes.

TABLE 1. Error table: $R T_{0}$ on structured mesh.

\begin{tabular}{lcccccc}
\hline Mesh size & \multicolumn{2}{c}{$\left\|\boldsymbol{\sigma}-\boldsymbol{\sigma}_{h}\right\|_{0}$} & \multicolumn{2}{c}{$\left\|\operatorname{div}\left(\boldsymbol{\sigma}-\boldsymbol{\sigma}_{h}\right)\right\|_{0}$} & \multicolumn{2}{c}{$\left\|P_{h} \varphi-\varphi_{h}\right\|_{0}$} \\
\hline & Err. & Order & Err. & Order & Err. & Order \\
\hline$h_{0}$ & 1.018359 & & 1.894110 & & 0.149686 & \\
$h_{0} / 2$ & 0.504852 & 1.01 & 0.902151 & 1.07 & 0.041618 & 1.84 \\
$h_{0} / 4$ & 0.251870 & 1.00 & 0.450850 & 1.00 & 0.010459 & 1.99 \\
$h_{0} / 8$ & 0.125915 & 1.00 & 0.225469 & 0.99 & 0.002625 & 1.99 \\
$h_{0} / 16$ & 0.062959 & 0.99 & 0.112742 & 0.99 & 0.000657 & 1.99 \\
\hline
\end{tabular}

TABLE 2. Error table: $R T_{0}$ on unstructured mesh.

\begin{tabular}{ccccccc}
\hline Mesh size & \multicolumn{2}{c}{$\left\|\boldsymbol{\sigma}-\boldsymbol{\sigma}_{h}\right\|_{0}$} & \multicolumn{2}{c}{$\left\|\operatorname{div}\left(\boldsymbol{\sigma}-\boldsymbol{\sigma}_{h}\right)\right\|_{0}$} & \multicolumn{2}{c}{$\left\|P_{h} \varphi-\varphi_{h}\right\|_{0}$} \\
\hline & Err. & Order & Err. & Order & Err. & Order \\
\hline$h_{0}$ & 0.583376 & & 0.750504 & & 0.063330 & \\
$h_{0} / 2$ & 0.294074 & 0.98 & 0.383364 & 0.96 & 0.016101 & 1.97 \\
$h_{0} / 4$ & 0.147403 & 0.99 & 0.192606 & 0.99 & 0.004053 & 1.98 \\
$h_{0} / 8$ & 0.073762 & 0.99 & 0.096416 & 0.99 & 0.001016 & 1.99 \\
\hline
\end{tabular}

\subsection{L-shaped domain}

In the second example we consider the non-convex and L-shaped domain $\Omega=\Omega_{0} \backslash \Omega_{1}$, where $\Omega_{0}$ is the square with side $\pi$, as before, and $\Omega_{1}$ is the square $\left(\frac{\pi}{2}, \pi\right) \times\left(\frac{\pi}{2}, \pi\right)$. Since $\Omega$ has a reentrant corner, eigenfunctions with singularities are expected. The numerical computations concern the first eigenfunction $(\boldsymbol{\sigma}, \varphi)$, which is singular and belongs to the space $H^{s}(\Omega)^{2} \times H^{1+s}(\Omega)$, with $s=\frac{2}{3}-\varepsilon, \forall \varepsilon>0$. Since no analytical expression for the exact eigenpair is known, in the numerical tests we take as exact eigenpair the one computed on a mesh fine enough.

We test the superconvergence property on a sequence of embedded meshes, which are obtained from an initial triangulation by regular refinement; Figure 5 shows the initial triangulation used for $R T_{0}$ and $B D M_{1}$ approximation, respectively. 

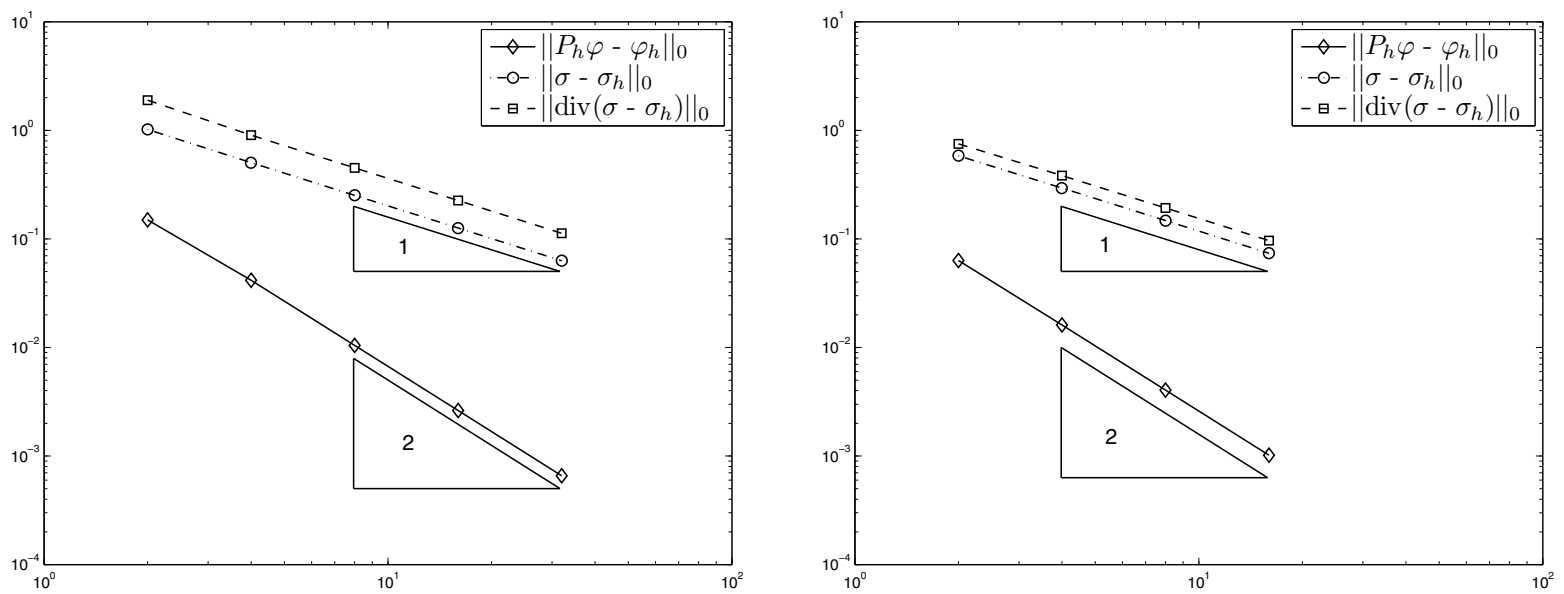

FIgURE 3. Errors versus $h^{-1}$ in $\log / \log$-scale for $R T_{0}$ on structured (left) and unstructured meshes (right).

TABLE 3. Error table: $B D M_{1}$ on structured mesh.

\begin{tabular}{lcccccc}
\hline Mesh size & \multicolumn{2}{c}{$\left\|\boldsymbol{\sigma}-\boldsymbol{\sigma}_{h}\right\|_{0}$} & \multicolumn{2}{c}{$\left\|\operatorname{div}\left(\boldsymbol{\sigma}-\boldsymbol{\sigma}_{h}\right)\right\|_{0}$} & \multicolumn{2}{c}{$\left\|P_{h} \varphi-\varphi_{h}\right\|_{0}$} \\
\hline & Err. & Order & Err. & Order & Err. & Order \\
\hline$h_{0}$ & 0.653615 & & 2.020459 & & 0.057680 & \\
$h_{0} / 2$ & 0.134918 & 2.27 & 0.921033 & 1.13 & 0.006028 & 3.25 \\
$h_{0} / 4$ & 0.032997 & 2.03 & 0.453273 & 1.02 & 0.002522 & 1.25 \\
$h_{0} / 8$ & 0.008265 & 1.99 & 0.225774 & 1.00 & 0.000712 & 1.82 \\
$h_{0} / 16$ & 0.002130 & 1.95 & 0.112780 & 1.00 & 0.000183 & 1.96 \\
\hline
\end{tabular}

TABLE 4. Error table: $B D M_{1}$ on unstructured mesh.

\begin{tabular}{lcccccc}
\hline Mesh size & \multicolumn{2}{c}{$\left\|\boldsymbol{\sigma}-\boldsymbol{\sigma}_{h}\right\|_{0}$} & \multicolumn{2}{c}{$\left\|\operatorname{div}\left(\boldsymbol{\sigma}-\boldsymbol{\sigma}_{h}\right)\right\|_{0}$} & \multicolumn{2}{c}{$\left\|P_{h} \varphi-\varphi_{h}\right\|_{0}$} \\
\hline & Err. & Order & Err. & Order & Err. & Order \\
\hline$h_{0}$ & 0.083746 & & 0.766087 & & 0.020439 & \\
$h_{0} / 2$ & 0.022797 & 1.87 & 0.385542 & 0.99 & 0.006066 & 1.75 \\
$h_{0} / 4$ & 0.005837 & 1.96 & 0.192885 & 0.99 & 0.001578 & 1.94 \\
$h_{0} / 8$ & 0.001553 & 1.91 & 0.096451 & 0.99 & 0.000398 & 1.98 \\
\hline
\end{tabular}

The first test concerns the lowest order Raviart-Thomas approximation. In Table 5 we report the $L^{2}$-norm of the error in the approximation of the eigenfunction and the $L^{2}$-norm of the error between the projection of the exact eigenfunction and the discrete one. We also compute the numerical rates of convergence, which agree with the ones predicted by the a priori error estimate (1.10) and by Theorem 3.2 for singular eigenmodes. Eventually, in Figure 6 we plot the above errors using a $\log / \log$ scale.

In the second test we consider $B D M_{1}$ finite elements. The results of these experiments are shown in Table 6 . The numerical rate of convergence agrees with the one predicted by the a priori error estimate (1.10) for singular eigenmodes. Moreover, the numerical results suggest that the superconvergence property holds as well. Finally, in Figure 7 we plot the errors in a $\log / \log$ scale. 

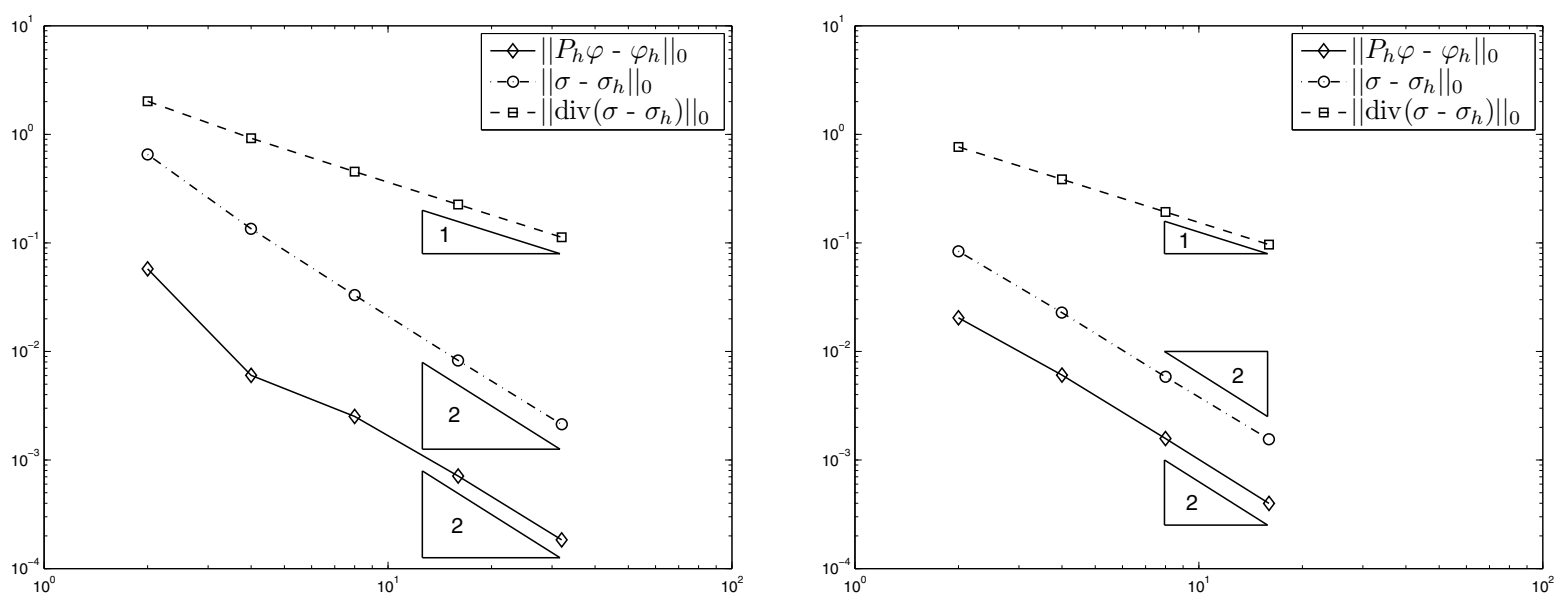

Figure 4. Errors versus $h^{-1}$ in $\log / \log$-scale for $B D M_{1}$ on structured (left) and unstructured meshes (right).
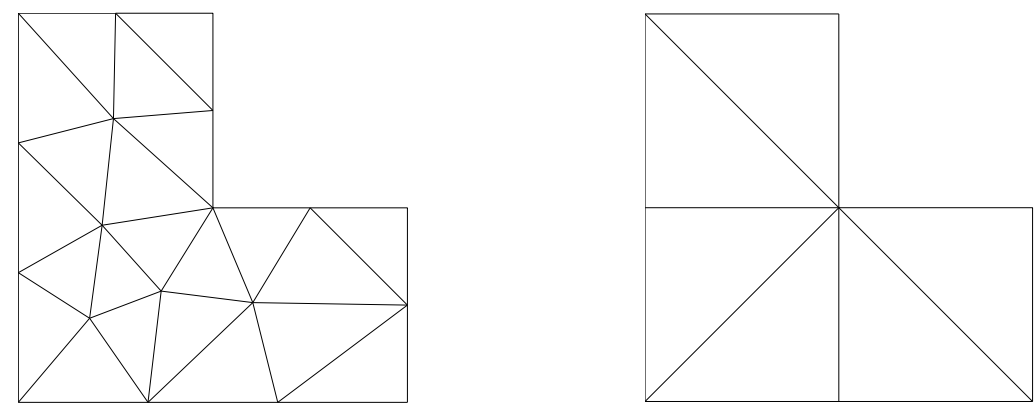

FIgURE 5. Initial triangulation for $R T_{0}$ (left) and $B D M_{1}$ (right) approximation.

TABLE 5. Error table: $R T_{0}$ on L-shaped domain.

\begin{tabular}{lcccc}
\hline Mesh size & \multicolumn{2}{c}{$\left\|\varphi-\varphi_{h}\right\|_{0}$} & \multicolumn{2}{c}{$\left\|P_{h} \varphi-\varphi_{h}\right\|_{0}$} \\
\hline & Err. & Order & Err. & Order \\
\hline$h_{0}$ & 0.200130 & & 0.078137 & \\
$h_{0} / 2$ & 0.100040 & 1.00 & 0.030291 & 1.36 \\
$h_{0} / 4$ & 0.050968 & 0.97 & 0.011840 & 1.35 \\
$h_{0} / 8$ & 0.028115 & 0.85 & 0.004660 & 1.34 \\
$h_{0} / 16$ & 0.018643 & 0.59 & 0.001843 & 1.33 \\
\hline
\end{tabular}

\section{Conclusions}

We stated a superconvergence result for the lowest order Raviart-Thomas approximation of Laplace eigenproblem. Numerical experiments confirm the superconvergence property and suggest that it also holds for the lowest order Brezzi-Douglas-Marini approximation.

For some possible application of this superconvergence result to a posteriori error estimates we refer to [12-14] and to forthcoming papers. 


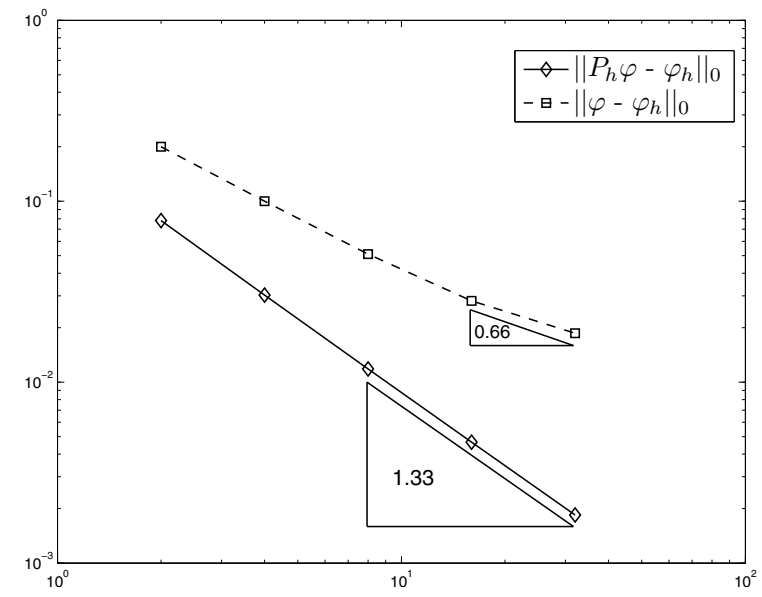

FiguRE 6. Errors versus $h^{-1}$ in $\log / \log$-scale for $R T_{0}$ on L-shaped domain.

TABLE 6. Error table: $B D M_{1}$ on L-shaped domain.

\begin{tabular}{lcccc}
\hline Mesh size & \multicolumn{1}{c}{$\left\|\varphi-\varphi_{h}\right\|_{0}$} & \multicolumn{2}{c}{$\left\|P_{h} \varphi-\varphi_{h}\right\|_{0}$} \\
\hline & Err. & Order & Err. & Order \\
\hline$h_{0}$ & 0.330278 & & 0.031675 & \\
$h_{0} / 2$ & 0.177920 & 0.89 & 0.015294 & 1.05 \\
$h_{0} / 4$ & 0.091043 & 0.96 & 0.005998 & 1.35 \\
$h_{0} / 8$ & 0.046512 & 0.96 & 0.002365 & 1.34 \\
$h_{0} / 16$ & 0.024683 & 0.91 & 0.000935 & 1.33 \\
$h_{0} / 32$ & 0.014793 & 0.73 & 0.000370 & 1.33 \\
\hline
\end{tabular}

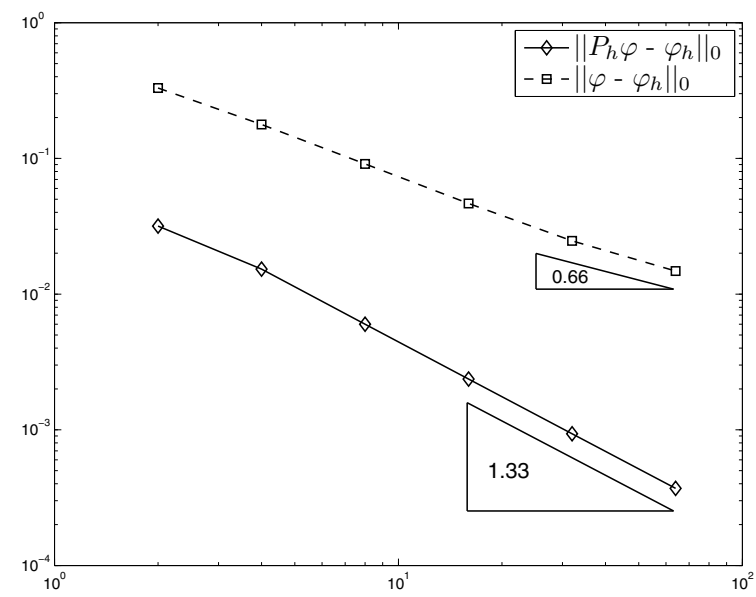

FiguRE 7 . Errors versus $h^{-1}$ in $\log / \log$-scale for $B D M_{1}$ on L-shaped domain.

Finally, possible future developments of the present work go towards the study of the superconvergence property for higher order elements and for general quadrilateral meshes. 


\section{REFERENCES}

[1] R.A. Adams, Sobolev spaces, Pure and Applied Mathematics 65. Academic Press, New York-London (1975).

[2] A. Alonso, A. Dello Russo and A. Vampa, A posteriori error estimates in finite element acoustic analysis. J. Comput. Appl. Math. 117 (2000) 105-119.

[3] A. Alonso, A. Dello Russo, C. Padra and R. Rodriguez, Accurate pressure post-process of a finite element method for elastoacoustics. Numer. Math. 98 (2004) 389-425.

[4] D.N. Arnold and F. Brezzi, Mixed and nonconforming finite element methods: implementation, postprocessing and error estimates. RAIRO Modél. Math. Anal. Numér. 19 (1985) 7-32.

[5] I. Babǔska and J. Osborn, Eigenvalue Problems, in Handbook of Numerical Analysis 2, P.G. Ciarlet and J.L. Lions Eds., North Holland (1991).

[6] D. Boffi, F. Brezzi and L. Gastaldi, On the convergence of eigenvalues for mixed formulations. Ann. Sc. Norm. Sup. Pisa Cl. Sci. 25 (1997) 131-154.

[7] D. Boffi, F. Kikuci and J. Schöberl, Edge element computation of Maxwell's eigenvalues on general quadrilateral meshes. Math. Models Methods Appl. Sci. 16 (2006) 265-273.

[8] J.H. Brandts, Superconvergence and a posteriori error estimation for triangular mixed finite elements. Numer. Math. 68 (1994) 311-324.

[9] F. Brezzi and M. Fortin, Mixed and hybrid finite element methods, Springer Series in Computational Mathematics 15. SpringerVerlag, New York (1991).

[10] P.G. Ciarlet, The finite element method for elliptic problems, Studies in Mathematics and its application 4. North Holland, Amsterdam (1978).

[11] R. Durán, L. Gastaldi and C. Padra, A posteriori error estimations for mixed approximation of eigenvalue problems. Math. Models Methods Appl. Sci. 9 (1999) 1165-1178.

[12] F. Gardini, A posteriori error estimates for eigenvalue problems in mixed form. Ist. lombardo Accd. Sci. Lett. Rend. A. 138 (2004) $17-34$.

[13] F. Gardini, A posteriori error estimates for an eigenvalue problem arising from fluid-structure interactions, Computational Fluid and Solid Mechanics. Elsevier, Amsterdam (2005).

[14] F. Gardini, A posteriori error estimates for eigenvalue problems in mixed form. Ph.D. Thesis, Università degli Studi di Pavia, Pavia, Italy (2005).

[15] P. Grisvard, Elliptic problem in nonsmooth domains, Monographs and Studies in Mathematics 24. Pitman, Boston (1985).

[16] J-.L. Lions and E. Magenes, Problèmes aux limites non homogènes et applications, Travaux et Recherches Mathematiques 17. Dunod, Paris (1968).

[17] L.D. Marini, An inexpensive method for the evaluation of the solution of the lowest order Raviart-Thomas mixed method. SIAM J. Numer. Anal. 22 (1985) 493-496. 